\title{
The Immunity of Non-Coherent and Coherent Reception of a Signal DQPSK in the Conditions of Influence of PSK, a Harmonic Interference or Gaussian Noise
}

\author{
Konstantin Yu. Lozhkin and Alexander I. Stitsenko* \\ Military Education and Research Centre of Military-Air Forces \\ «Military-Air Academy \\ Named After Professor N.E. Zhukovsky and Yu.A. Gagarin» \\ 54a Starykh Bolshevikov Str., Voronezh, 394064, Russia
}

Received 23.10.2017, received in revised form 25.12.2017, accepted 19.01.2017

By the method of making the indicators of output elements obtained according to the average probability of correct reception in optimum incoherent receiving-solver symbol and binary element no fading DQPSK signal when exposed to PSK or harmonic interference from the signal-to-noise ratio. The analysis of these dependencies and comparison of optimal noise immunity of incoherent and coherent of receivers with DQPSK signal with PSK, a harmonic interference and Gaussian noise.

Keywords: DQPSK signal, optimal incoherent receiving-solver, PSK, harmonic interference, Gaussian noise, probabilistic-surface indicators of reliability of signal reception.

Citation: Lozhkin K.Yu., Stitsenko A.I. The immunity of non-coherent and coherent reception of a signal DQPSK in the conditions of influence of PSK, a harmonic interference or Gaussian noise, J. Sib. Fed. Univ. Eng. technol., 2017, $10(2), 260$ 270. DOI: 10.17516/1999-494X-2017-10-2-260-270.

(C) Siberian Federal University. All rights reserved

* Corresponding author E-mail address: K.Yu.Lozhkin@mail.ru, sticenko@mail.ru 


\title{
Помехоустойчивость некогерентного и когерентного приема ДФРМ-сигнала в условиях воздействия
} фазоманипулированной, гармонической или гауссовской помех

\author{
К.Ю. Ложкин, А.И. Стиценко \\ Военный учебно-научный центр Военно-воздушных сил \\ «Военно-воздушная академия \\ имени профессора Н.Е. Жуковского и Ю.А. Гагарина» \\ Россия, 394064, Воронеж, ул. Старых Большевиков, 54 а
}

\begin{abstract}
Методом индикаторов решений о переданных элементах получены зависимости средних вероятностей правильного приема в оптимальном некогерентном приемно-решающем устройстве символа и двоичного элемента простого незамирающего сигнала с двукратной фазоразностной манипуляцией при воздействии фазоманипулированной или гармонической помех от отномения сигнал-помеха. Проведен анализ указанных зависимостей и выполнено сравнение помехоустойчивости оптимального некогерентного и когерентного приемнорешающих устройств сигналов с двукратной фазоразностной манипулящией при воздействии фазоманипулированной, гармонической и гауссовской помех.

Ключевые слова: сигнал с двукратной фазоразностной манипуляцией; оптимальное некогерентное приемно-решающее устройство; фазоманипулированная, гармоническая, гауассовская помехи; вероятностностные показатели достоверности приема сигнала.
\end{abstract}

\section{Актуальность и цель работы}

В практике радиосвязи широкое применение находят сигналы с двукратной фазоразностной манипуляцией (ДФРМ), прием которых осуществляется некогерентными и когерентными приемно-решающими устройствами (ПРУ) [1]. Ряд задач оценки эффективности конфликтного взаимодействия линий радиосвязи, использующих такие сигналы, и источников станционных и преднамеренных помех решаются с применением методик расчета средних вероятностей правильного приема символа и двоичного элемента в ПРУ. Известны результаты исследований помехоустойчивости когерентного приема ДФРМ-сигнала в условиях гауссовской и незамирающей гармонической помех [2-4] и некогерентного приема этого сигнала при воздействии гауссовской помехи [1]. Однако в литературе отсутствуют результаты сравнительной оценки помехоустойчивости указанных ПРУ при воздействии фазоманипулированной и гармонической помех, являющихся удовлетворительными моделями сигналоподобных преднамеренных и непреднамеренных помеховых воздействий. Поэтому актуальна задача оценки помехоустойчивости оптимального некогерентного и когерентного приема ДФРМ-сигнала в условиях воздействия фазоманипулированной и гармонической помех, а также сравнительный анализ полученных результатов с известными [1-3] в части помехоустойчивости рассматриваемых ПРУ в условиях гауссовской помехи.

Цель работы - получение зависимостей средних вероятностей правильного приема в оптимальном некогерентном ПРУ символа и двоичного элемента простого незамирающего ДФРМ-сигнала в условиях воздействия фазоманипулированной или гармонической помехи от отношения сигнал-помеха, а также сравнительный анализ помехоустойчивости оптималь- 
ного некогерентного и когерентного ПРУ при воздействии перечисленных и гауссовской поMex.

\section{Постановка задачи}

Поставленную задачу решим методом индикаторов решений о переданных элементах $[5,6]$ в предположении, что прием аддитивной смеси сигнала и помехи осуществляется оптимальным некогерентным ПРУ [1, с. 171]. Положим, что мощность внутренних и внешних шумов пренебрежимо мала по сравнению с мощностью сигнала воздействующей помехи.

Рассматриваемый сигнал на интервале приема $(n-1)$-й («опорной») и $n$-й («текущей») посылок запишем в следующем виде:

$$
\begin{aligned}
& s_{n-1}(t)=U_{\mathrm{s}} \sin \left(\omega_{\mathrm{s}} t+\varphi_{\mathrm{S}}+\frac{\pi}{4}+\Theta_{n-1}\right), \quad(n-1) T<t \leq n T, \\
& s_{n}(t)=U_{\mathrm{s}} \sin \left(\omega_{\mathrm{s}} t+\varphi_{\mathrm{S}}+\frac{\pi}{4}+\Theta_{n}\right), \quad n T<t \leq(n+1) T,
\end{aligned}
$$

где $U_{s}, \omega_{s}, T, \varphi_{s}$ - амплитуда, несущая частота, длительность посылки и начальная фаза сигнала, которую можно считать постоянной величиной на интервале $(n-1) T<t \leq(n+1) T ; \Theta_{n-1}$ и $\Theta_{n}-$ приращения фазы сигнала. Разность фаз $\Delta \Theta_{n}=\Theta_{n}-\Theta_{n-1}$ соседних посылок сигнала (1) содержит информацию о передаваемых двоичных элементах $i_{n}=0,1$ и $q_{n}=0,1$. Соответствие величин $i_{n}, q_{n}, \Delta \Theta_{n}, \Theta_{n-1}, \Theta_{n}$ и напряжений $I_{n}, Q_{n}$ на выходе ПРУ при использовании манипуляционного кода Грея приведены в табл. [1, с. 169].

Таблица. Соответствие значений передаваемых двоичных элементов, разности фаз соседних посылок

\begin{tabular}{|c|c|c|c|c|c|c|}
\hline \multicolumn{2}{|c|}{$\begin{array}{c}\text { Передаваемые } \\
\text { двоичные } \\
\text { элементы }\end{array}$} & \multirow[t]{2}{*}{$\begin{array}{c}\text { Разность фаз } \\
\text { соседних } \\
\text { посылок } \\
\text { сигнала } \Delta \Theta_{n}\end{array}$} & \multicolumn{2}{|c|}{$\begin{array}{c}\text { Значения приращения } \\
\text { фазы сигнала }\end{array}$} & \multicolumn{2}{|c|}{$\begin{array}{c}\text { Напряжения на выходе } \\
\text { каналов ПРУ в случае } \\
\text { принятия правильных } \\
\text { решений }\end{array}$} \\
\hline$\overline{i_{n}}$ & $q_{n}$ & & $\Theta_{n-1}$ & $\Theta_{n}$ & $I_{n}$ & $Q_{n}$ \\
\hline \multirow{4}{*}{0} & \multirow{4}{*}{0} & \multirow{4}{*}{0} & 0 & 0 & \multirow{4}{*}{+} & \multirow{4}{*}{+} \\
\hline & & & $\pi / 2$ & $\pi / 2$ & & \\
\hline & & & $\pi$ & $\pi$ & & \\
\hline & & & $3 \pi / 2$ & $3 \pi / 2$ & & \\
\hline \multirow{4}{*}{0} & \multirow{4}{*}{1} & \multirow{4}{*}{$\pi / 2$} & 0 & $\pi / 2$ & \multirow{4}{*}{+} & \multirow{4}{*}{-} \\
\hline & & & $\pi / 2$ & $\pi$ & & \\
\hline & & & $\pi$ & $3 \pi / 2$ & & \\
\hline & & & $3 \pi / 2$ & 0 & & \\
\hline \multirow{4}{*}{1} & \multirow{4}{*}{1} & \multirow{4}{*}{$\pi$} & 0 & $\pi$ & \multirow{4}{*}{-} & \multirow{4}{*}{-} \\
\hline & & & $\pi / 2$ & $3 \pi / 2$ & & \\
\hline & & & $\pi$ & 0 & & \\
\hline & & & $3 \pi / 2$ & $\pi / 2$ & & \\
\hline \multirow{4}{*}{1} & \multirow{4}{*}{0} & \multirow{4}{*}{$3 \pi / 2$} & 0 & $3 \pi / 2$ & \multirow{4}{*}{-} & \multirow{4}{*}{+} \\
\hline & & & $\pi / 2$ & 0 & & \\
\hline & & & $\pi$ & $\pi / 2$ & & \\
\hline & & & $3 \pi / 2$ & $\pi$ & & \\
\hline
\end{tabular}
сигнала и напряжений на выходе ПРУ 
Фазоманипулированную помеху (ФМП), частота и тактовые моменты манипуляции которой совпадают с соответствующими параметрами сигнала (1) на входе ПРУ, на интервале приема $(n-1)$-й и $n$-й посылок сигнала представим в виде

$$
\begin{array}{ll}
u_{n-1}(t)=U_{\mathrm{j}} \sin \left(\omega_{\mathrm{s}} t+\varphi_{n-1}\right), & (n-1) T<t \leq n T, \\
u_{n}(t)=U_{\mathrm{j}} \sin \left(\omega_{\mathrm{s}} t+\varphi_{n}\right), & n T<t \leq(n+1) T,
\end{array}
$$

где $U_{j}$ - амплитуда ФМП; $\varphi_{n-1}, \varphi_{n}$ - ее начальные фазы, являющиеся независимыми равномерно распределенными от 0 до $2 \pi$ случайными величинами.

Аналогично на интервале приема $(n-1)$-й и $n$-й посылок сигнала (1) запишем выражение для гармонической помехи (ГП) с амплитудой $U_{j}$

$$
u_{\mathrm{hi}}(t)=U_{\mathrm{j}} \sin \left(\omega_{\mathrm{s}} t+\varphi_{\mathrm{hi}}\right), \quad(n-1) T<t \leq(n+1) T,
$$

частота которой совпадает с частотой сигнала, а начальная фаза $\varphi_{h i}$ является равномерно распределенной от 0 до $2 \pi$ случайной величиной, изменяющейся от сеанса к сеансу связи.

Для решения поставленной задачи вначале конкретизируем для рассматриваемых помех напряжения $f_{I}$ и $f_{Q}$ на входах решающих устройств ПРУ:

$$
\begin{aligned}
& f_{I}=\left(X_{n} X_{n-1}+Y_{n} Y_{n-1}\right)-\left(Y_{n} X_{n-1}-X_{n} Y_{n-1}\right), \\
& f_{Q}=\left(X_{n} X_{n-1}+Y_{n} Y_{n-1}\right)+\left(Y_{n} X_{n-1}-X_{n} Y_{n-1}\right),
\end{aligned}
$$

где $X_{n-1}, X_{n}$ и $Y_{n-1}, Y_{n}$ - напряжения на выходах корреляторов синфазного и квадратурного каналов в моменты окончания $(n-1)$-й и $n$-й посылок сигнала. Указанные напряжения в соответствии со структурной схемой ПРУ [1, с. 171] описываются следующими выражениями: для ФМП (2)

для ГП (3)

$$
\begin{aligned}
& X_{n-1}=\frac{1}{T} \int_{(n-1) T}^{n T}\left[s_{n-1}(t)+u_{n-1}(t)\right] u_{I}(t), \quad X_{n}=\frac{1}{T} \int_{n T}^{(n+1) T}\left[s_{n}(t)+u_{n}(t)\right] u_{I}(t), \\
& Y_{n-1}=\frac{1}{T} \int_{(n-1) T}^{n T}\left[s_{n-1}(t)+u_{n-1}(t)\right] u_{Q}(t), \quad Y_{n}=\frac{1}{T} \int_{n T}^{(n+1) T}\left[s_{n}(t)+u_{n}(t)\right] u_{Q}(t) ;
\end{aligned}
$$

$$
\begin{gathered}
X_{n-1}=\frac{1}{T} \int_{(n-1) T}^{n T}\left[s_{n-1}(t)+u_{\mathrm{hi}}(t)\right] u_{I}(t), \quad X_{n}=\frac{1}{T} \int_{n T}^{(n+1) T}\left[s_{n}(t)+u_{\mathrm{hi}}(t)\right] u_{I}(t), \\
Y_{n-1}=\frac{1}{T} \int_{(n-1) T}^{n T}\left[s_{n-1}(t)+u_{\mathrm{hi}}(t)\right] u_{Q}(t), \quad Y_{n}=\frac{1}{T} \int_{n T}^{(n+1) T}\left[s_{n}(t)+u_{\mathrm{hi}}(t)\right] u_{Q}(t),
\end{gathered}
$$

где $u_{I}(t)=\sin \left(\omega_{\mathrm{s}} t\right), u_{Q}(t)=\sin \left(\omega_{\mathrm{s}} t+\pi / 2\right)$ - опорные напряжения корреляторов синфазного и квадратурного каналов.

Напряжения $f_{I}$ и $f_{Q}$ для помех (2), (3) получим следующим образом.

Для ФМП - путем подстановки (1) и (2) в (5), а (5) - в (4):

$$
\begin{gathered}
f_{I}\left(h_{\mathrm{S}}, \Theta_{n-1}, \Theta_{n}, \varphi_{n-1}, \varphi_{n}, \varphi_{\mathrm{s}}\right)= \\
\quad=h_{\mathrm{S}}^{2} \cos \left[\frac{\pi}{4}-\left(\Theta_{n}-\Theta_{n-1}\right)\right]+\cos \left[\frac{\pi}{4}-\left(\varphi_{n}-\varphi_{n-1}\right)\right]+h_{\mathrm{S}} \cos \left(\frac{\pi}{2}-\varphi_{n}+\varphi_{\mathrm{s}}+\Theta_{n-1}\right)+h_{\mathrm{s}} \cos \left(\varphi_{n-1}-\varphi_{\mathrm{s}}-\Theta_{n}\right), \\
f_{Q}\left(h_{\mathrm{s}}, \Theta_{n-1}, \Theta_{n}, \varphi_{n-1}, \varphi_{n}, \varphi_{\mathrm{s}}\right)= \\
=h_{\mathrm{S}}^{2} \cos \left[\frac{\pi}{4}+\left(\Theta_{n}-\Theta_{n-1}\right)\right]+\cos \left[\frac{\pi}{4}+\left(\varphi_{n}-\varphi_{n-1}\right)\right]+h_{\mathrm{S}} \cos \left(\varphi_{n}-\varphi_{\mathrm{s}}-\Theta_{n-1}\right)+h_{\mathrm{s}} \cos \left(\frac{\pi}{2}-\varphi_{n-1}+\varphi_{\mathrm{S}}+\Theta_{n}\right) . \\
-263-
\end{gathered}
$$


Для ГП - путем подстановки (1) и (3) - в (6), а затем (6) - в (4):

$$
\begin{aligned}
& f_{I}\left(h_{\mathrm{s}}, \Theta_{n-1}, \Theta_{n}, \varphi_{\mathrm{hi}}, \varphi_{\mathrm{s}}\right)=h_{\mathrm{s}}^{2} \cos \left[\frac{\pi}{4}-\left(\Theta_{n}-\Theta_{n-1}\right)\right]+h_{\mathrm{s}} \cos \left(\frac{\pi}{2}-\varphi_{\mathrm{hi}}+\varphi_{\mathrm{s}}+\Theta_{n-1}\right)+h_{\mathrm{s}} \cos \left(\varphi_{\mathrm{hi}}-\varphi_{\mathrm{s}}-\Theta_{n}\right)+\frac{\sqrt{2}}{2}, \\
& f_{Q}\left(h_{\mathrm{s}}, \Theta_{n-1}, \Theta_{n}, \varphi_{\mathrm{hi}}, \varphi_{\mathrm{s}}\right)=h_{\mathrm{s}}^{2} \cos \left[\frac{\pi}{4}+\left(\Theta_{n}-\Theta_{n-1}\right)\right]+h_{\mathrm{s}} \cos \left(\varphi_{\mathrm{hi}}-\varphi_{\mathrm{s}}-\Theta_{n-1}\right)+h_{\mathrm{s}} \cos \left(\frac{\pi}{2}-\varphi_{\mathrm{hi}}+\varphi_{\mathrm{s}}+\Theta_{n}\right)+\frac{\sqrt{2}}{2}
\end{aligned}
$$

где $h_{\mathrm{S}}=U_{\mathrm{S}} / U_{\mathrm{j}}$ - отношение сигнал-помеха по напряжению на входе ПРУ.

Далее, следуя методу индикаторов решений о переданных элементах [5, 6], запишем правила принятия решений $\hat{i}_{n}, \hat{q}_{n}$ о переданных двоичных элементах $i_{n}, q_{n}$, индикаторы правильных решений об этих элементах $I_{i}, I_{q}$, а также выражения средних вероятностей правильного приема символа $\dot{p}_{\mathrm{s}}$ и двоичного элемента $\dot{p}_{\mathrm{b}}$ ДФРМ сигнала в рассматриваемом ПРУ при воздействии ФМП или ГП.

Вначале приведем указанные соотношения в общем виде, а затем конкретизируем их применительно к воздействию ФМП (2) и ГП (3).

\section{Решение задачи}

Правила принятия решений $\hat{i}_{n}, \hat{q}_{n}$ о переданных двоичных элементах $i_{n}, q_{n}$ с учетом таблицы для рассматриваемого ПРУ описываются неравенствами

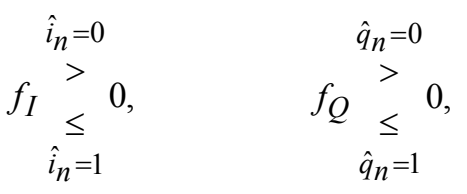

где $f_{I}$ и $f_{Q}$ определяются выражениями (7) для ФМП и (8) для ГП.

Индикаторы $I_{i}, I_{q}$ событий [5], заключающихся в правильном принятии решений о переданных двоичных элементах $i_{n}, q_{n}$, на основании правил (9), выражений (7), (8) и приведенного в таблице соответствия всех возможных значений величин $\Theta_{n-1}, \Theta_{n}$ и $\Delta \Theta_{n}$ представим следующим образом:

$$
\begin{array}{ll}
\text { при } \Delta \Theta_{n}=\Theta_{n}-\Theta_{n-1}=\{0 ; \pi / 2\}, & I_{i}=\left\{\begin{array}{lll}
1, & \text { если } & f_{I}>0, \\
0, & \text { если } & f_{I} \leq 0 ;
\end{array}\right. \\
\text { при } \Delta \Theta_{n}=\{\pi ; 3 \pi / 2\}, & I_{i}=\left\{\begin{array}{lll}
1, & \text { если } & f_{I}<0, \\
0, & \text { если } & f_{I} \geq 0 ;
\end{array}\right. \\
\text { при } \Delta \Theta_{n}=\{0 ; 3 \pi / 2\}, & I_{q}=\left\{\begin{array}{lll}
1, & \text { если } & f_{I}>0, \\
0, & \text { если } & f_{I} \leq 0 ;
\end{array}\right. \\
\text { при } \Delta \Theta_{n}=\{\pi / 2 ; \pi\}, & I_{q}=\left\{\begin{array}{lll}
1, & \text { если } & f_{I}<0, \\
0, & \text { если } & f_{I} \geq 0 .
\end{array}\right.
\end{array}
$$

При получении средних вероятностей правильного приема символа $\dot{p}_{\mathrm{s}}$ и двоичного элемента $\dot{p}_{\mathrm{b}}$ ДФРМ сигнала положим, что все возможные значения $0, \frac{\pi}{2}, \pi, \frac{3 \pi}{2}$ величин $\Theta_{n-1}$, $\Theta_{n}$ появляются независимо и равновероятно. Поэтому при получении выражений $\dot{p}_{\mathrm{s}}$ и $\dot{p}_{\mathrm{b}}$ применим формулу полной вероятности и учтем, что вероятность появления события $Z$ равна ма- 
тематическому ожиданию $\mathrm{M}\left\{I_{z}\right\}$ индикатора $I_{z}$ этого события, а индикатор пересечения событий равен произведению индикаторов этих событий [5].

На основании изложенного среднюю вероятность правильного приема символа сигнала (1) при воздействии помехи запишем в обобщенном виде:

$$
\dot{p}_{\mathrm{S}}=\sum_{k=1}^{4} \sum_{\ell=1}^{4} P\left\{\Theta_{[(n-1), \ell]}\right\} P\left\{\Theta_{(n, k)}\right\} \dot{P}\left\{\left(\hat{i}_{n}=i_{n}\right) \cap\left(\hat{q}_{n}=q_{n}\right) \mid \Theta_{[(n-1), \ell]}, \Theta_{(n, k)}\right\}
$$

где величины $\Theta_{[(n-1), \ell]}, \ell=\overline{1,4}, \Theta_{(n, k)}, k=\overline{1,4}$, независимо принимают значения $0, \frac{\pi}{2}, \pi, \frac{3 \pi}{2}$ с вероятностями $P\left\{\Theta_{[(n-1), \ell]}\right\}=P\left\{\Theta_{(n, k)}\right\}=1 / 4, \dot{P}\left\{\left(\hat{i}_{n}=i_{n}\right) \cap\left(\hat{q}_{n}=q_{n}\right) \mid \Theta_{[(n-1), \ell]}, \Theta_{(n, k)}\right\}-$ вероятность правильного приема символа ДФРМ сигнала (одновременного правильного приема двоичных элементов $i_{n}$ и $q_{n}$ ) при условии появления конкретных значений $\Theta_{[(n-1), \ell]}$ и $\Theta_{(n, k)}$.

Аналогично получим обобщенное выражение средней вероятности правильного приема двоичного элемента ДФРМ сигнала

$$
\begin{aligned}
& \dot{p}_{\mathrm{b}}=\frac{1}{2}\left[\dot{P}\left\{\hat{i}_{n}=i_{n}\right\}+\dot{P}\left\{\hat{q}_{n}=q_{n}\right\}\right]= \\
& =\frac{1}{2}\left[\sum_{k=1}^{4} \sum_{\ell=1}^{4} P\left\{\Theta_{[(n-1), \ell]}\right\} P\left\{\Theta_{(n, k)}\right) \dot{P}\left\{\left(\hat{i}_{n}=i_{n}\right) \mid \Theta_{[(n-1), \ell]}, \Theta_{(n, k)}\right\}+\right. \\
& \left.\quad+\sum_{k=1}^{4} \sum_{j=1}^{4} P\left\{\Theta_{[(n-1), \ell]}\right\} P\left\{\Theta_{(n, k)}\right\} \dot{P}\left\{\left(\hat{q}_{n}=q_{n}\right) \mid \Theta_{[(n-1), \ell]}, \Theta_{(n, k)}\right\}\right],
\end{aligned}
$$

где $\dot{P}\left\{\hat{i}_{n}=i_{n}\right\}, \dot{P}\left\{\hat{q}_{n}=q_{n}\right\}$ - средние вероятности правильного приема двоичных элементов $i_{n}$ и $q_{n}, \dot{P}\left\{\left(\hat{i}_{n}=i_{n}\right) \Theta_{[(n-1), \ell]}, \Theta_{(n, k)}\right\}, \dot{P}\left\{\left(\hat{q}_{n}=q_{n}\right) \Theta_{[(n-1), \ell]}, \Theta_{(n, k)}\right\}$ - вероятности правильного приема двоичных элементов $i_{n}$ и $q_{n}$ при условии появления конкретных значений $\Theta_{[(n-1), \ell]}$ и $\Theta_{(n, k)}$.

Конкретизируем выражения (11), (12) вероятностей $\dot{p}_{\mathrm{s}}$ и $\dot{p}_{\mathrm{b}}$ для случая воздействия на рассматриваемое ПРУ ФМП со случайным набегом фазы (2). При этом учтем, что согласно (7) и (9) индикаторы $I_{i}, I_{q}(10)$ для этой помехи являются функциями величин $h_{s}, \Theta_{n-1}, \Theta_{n}, \varphi_{n-1}, \varphi_{n}$, $\varphi_{s}: I_{i}=I_{i}\left(h_{\mathrm{s}}, \Theta_{n-1}, \Theta_{n}, \varphi_{n-1}, \varphi_{n}, \varphi_{\mathrm{s}}\right), I_{q}=I_{q}\left(h_{\mathrm{s}}, \Theta_{n-1}, \Theta_{n}, \varphi_{n-1}, \varphi_{n}, \varphi_{\mathrm{s}}\right)$.

Подставим выражения $f_{I}$ и $f_{Q}(7)$ в (10) при соответствующих значениях $\Theta_{n-1}, \Theta_{n}$ и $\Delta \Theta_{n}$ (см. табл.), а выражения (10) - в (11) и (12) и учтем, что плотности вероятности независимых случайных величин $\varphi_{n-1}$ и $\varphi_{n}$ определяюстя как $W\left(\varphi_{n-1}\right)=W\left(\varphi_{n}\right)=1 /(2 \pi), 0 \leq \varphi_{n-1} \leq 2 \pi$, $0 \leq \varphi_{n} \leq 2 \pi$.

После выполненных подстановок в результете численных расчетов можно показать, что при воздействии на рассматриваемое ПРУ помехи (2) входящие в (11) условные вероятности

$$
\begin{aligned}
\dot{P}\left\{\left(\hat{i}_{n}=\right.\right. & \left.\left.i_{n}\right) \cap\left(\hat{q}_{n}=q_{n}\right) \mid \Theta_{[(n-1), \ell]}, \Theta_{(n, k)}\right\}= \\
& =\iint\left[I_{i}\left(h_{\mathrm{c}}, \Theta_{n-1}, \Theta_{n}, \varphi_{n-1}, \varphi_{n}, \varphi_{\mathrm{c}}\right) I_{q}\left(h_{\mathrm{c}}, \Theta_{n-1}, \Theta_{n}, \varphi_{n-1}, \varphi_{n}, \varphi_{\mathrm{c}}\right)\right] W\left(\varphi_{n-1}\right) W\left(\varphi_{n}\right) d \varphi_{n-1} d \varphi_{n},
\end{aligned}
$$

равны при всех возможных значениях $0, \frac{\pi}{2}, \pi, \frac{3 \pi}{2}$ величин $\Theta_{[(n-1), \ell]}, \ell=\overline{1,4}, \Theta_{(n, k)}, k=\overline{1,4}$.

Поэтому выражение вероятности $\dot{p}_{\mathrm{s}}(11)$ применительно к воздействию ФМП (2) принимает следующий вид: 


$$
\begin{aligned}
& \dot{p}_{\mathrm{s}}=\dot{P}\left\{\left(\hat{i}_{n}=i_{n}\right) \cap\left(\hat{q}_{n}=q_{n}\right) \mid \Theta_{[(n-1), 1]}, \Theta_{(n, 1)}\right\}= \\
& =\frac{1}{(2 \pi)^{2}} \int_{0}^{2 \pi} \int_{0}^{2 \pi}\left[I_{i}\left(h_{\mathrm{s}}, \Theta_{n-1}, \Theta_{n}, \varphi_{n-1}, \varphi_{n}, \varphi_{\mathrm{s}}\right) I_{q}\left(h_{\mathrm{s}}, \Theta_{n-1}, \Theta_{n}, \varphi_{n-1}, \varphi_{n}, \varphi_{\mathrm{s}}\right)\right] \\
& \|_{n-1}=0, \Theta_{n}=0 \\
& \Theta_{n-1} d \varphi_{n} .
\end{aligned}
$$

Также с применением численных расчетов можно показать, что при воздействии помехи (2) входящие в (12) условные вероятности

$$
\dot{P}\left\{\left(\hat{i}_{n}=i_{n}\right) \mid \Theta_{[(n-1), \ell]}, \Theta_{(n, k)}\right\}=\int_{0}^{2 \pi} \int_{0}^{2 \pi}\left[I_{i}\left(h_{\mathrm{s}}, \Theta_{n-1}, \Theta_{n}, \varphi_{n-1}, \varphi_{n}, \varphi_{\mathrm{s}}\right) W\left(\varphi_{n-1}\right) W\left(\varphi_{n}\right) d \varphi_{n-1} d \varphi_{n}\right.
$$

и

$$
\dot{P}\left\{\left(\hat{q}_{n}=q_{n}\right) \mid \Theta_{[(n-1), \ell]}, \Theta_{(n, k)}\right\}=\int_{0}^{2 \pi} \int_{0}^{2 \pi}\left[I_{q}\left(h_{\mathrm{s}}, \Theta_{n-1}, \Theta_{n}, \varphi_{n-1}, \varphi_{n}, \varphi_{\mathrm{s}}\right)\right] W\left(\varphi_{n-1}\right) W\left(\varphi_{n}\right) d \varphi_{n-1} d \varphi_{n}
$$

равны при всех возможных значениях $0, \frac{\pi}{2}, \pi, \frac{3 \pi}{2}$ величин $\Theta_{[(n-1), \ell]}, \ell=\overline{1,4}, \Theta_{(n, k)}, k=\overline{1,4}$.

Поэтому выражение вероятности $\dot{p}_{\mathrm{b}}(12)$ для случая воздействия ФМП (2) запишем следующим образом:

$$
\dot{p}_{\mathrm{b}}=\frac{1}{(2 \pi)^{2}} \int_{0}^{2 \pi} \int_{0}^{2 \pi} I_{i}\left(h_{\mathrm{s}}, \Theta_{n-1}, \Theta_{n}, \varphi_{n-1}, \varphi_{n}, \varphi_{\mathrm{s}}\right)_{\Theta_{n-1}=0, \Theta_{n}=0} d \varphi_{n-1} d \varphi_{n} .
$$

Важно отметить, что, как и следовало ожидать для некогерентного ПРУ, средние вероятности правильного приема символа и двоичного элемента ДФРМ-сигнала при воздействии фазоманипулированной помехи не зависят от начальной фазы сигнала $\varphi_{s}$. Действительно, изменение значения $\varphi_{s}$ в интегралах при расчете вероятностей (13), (14) смещает подынтегральную функцию в области определения, но не меняет значений указанных вероятностей.

С использованием симметрии подынтегральных функций в выражениях вероятностей (13) и (14) относительно плоскостей, описываемых уравнениями $\varphi_{n-1}=\left(2 \pi-\varphi_{n}\right)$ и $\varphi_{n-1}=\varphi_{n}$ соответственно, возможно сокращение временных затрат на численные расчеты путем применения эквивалентных выражений с уменьшенной вдвое областью интегрирования:

$$
\begin{gathered}
\dot{p}_{\mathrm{s}}=\frac{1}{2 \pi^{2}} \int_{0}^{2 \pi} \int_{0}^{2 \pi-\varphi_{n}}\left[I_{i}\left(h_{\mathrm{s}}, \Theta_{n-1}, \Theta_{n}, \varphi_{n-1}, \varphi_{n}, \varphi_{\mathrm{s}}\right) \times\right. \\
\left.\quad \times I_{q}\left(h_{\mathrm{s}}, \Theta_{n-1}, \Theta_{n}, \varphi_{n-1}, \varphi_{n}, \varphi_{\mathrm{s}}\right)\right]_{\Theta_{n-1}=0, \Theta_{n}=0} d \varphi_{n-1} d \varphi_{n}, \\
\dot{p}_{\mathrm{b}}=\frac{1}{2 \pi^{2}} \int_{0}^{2 \pi} \int_{0}^{\varphi_{n}} I_{i}\left(h_{\mathrm{s}}, \Theta_{n-1}, \Theta_{n}, \varphi_{n-1}, \varphi_{n}, \varphi_{\mathrm{s}}\right)_{\Theta_{n-1}=0, \Theta_{n}=0} d \varphi_{n-1} d \varphi_{n} .
\end{gathered}
$$

При получении выражений вероятностей $\dot{p}_{\mathrm{s}}$ и $\dot{p}_{\mathrm{b}}$ для случая воздействия на ПРУ ДФРМсигналов ГП (3) с нулевой расстройкой по частоте отностительно сигнала учтем, что согласно (8) и (9) индикаторы $I_{i}, I_{q}(10)$ для этой помехи являются функциями величин $h_{s}, \Theta_{n-1}, \Theta_{n}, \varphi_{h i}, \varphi_{s}$ : $I_{i}=I_{i}\left(h_{\mathrm{s}}, \Theta_{n-1}, \Theta_{n}, \varphi_{\mathrm{hi}}, \varphi_{\mathrm{s}}\right), I_{q}=I_{q}\left(h_{\mathrm{s}}, \Theta_{n-1}, \Theta_{n}, \varphi_{\mathrm{hi}}, \varphi_{\mathrm{s}}\right)$.

$$
-266-
$$


С применением выражений индикаторов $I_{i}\left(h_{\mathrm{s}}, \Theta_{n-1}, \Theta_{n}, \varphi_{\mathrm{hi}}, \varphi_{\mathrm{s}}\right), I_{q}\left(h_{\mathrm{s}}, \Theta_{n-1}, \Theta_{n}, \varphi_{\mathrm{hi}}, \varphi_{\mathrm{s}}\right)$ (10) из выражений (11) и (12) получим вероятности $\dot{p}_{\mathrm{s}}$ и $\dot{p}_{\mathrm{b}}$ для случая воздействия на рассматриваемое ПРУ помехи (3):

$$
\begin{gathered}
\dot{p}_{\mathrm{s}}=\frac{1}{16} \sum_{k=1}^{4} \sum_{\ell=1}^{4} \mathrm{M}_{\varphi_{\mathrm{hi}}}\left\{I_{i}\left(h_{\mathrm{s}}, \Theta_{[(n-1), \ell]}, \Theta_{(n, k)}, \varphi_{\mathrm{hi}}, \varphi_{\mathrm{s}}\right) I_{q}\left(h_{\mathrm{s}}, \Theta_{[(n-1), \ell]}, \Theta_{(n, k)}, \varphi_{\mathrm{hi}}, \varphi_{\mathrm{s}}\right)\right\}= \\
=\frac{1}{16} \sum_{k=1}^{4} \sum_{\ell=1}^{4} \int_{0}^{2 \pi} I_{i}\left(h_{\mathrm{s}}, \Theta_{[(n-1), \ell]}, \Theta_{(n, k)}, \varphi_{\mathrm{hi}}, \varphi_{\mathrm{s}}\right) I_{q}\left(h_{\mathrm{s}}, \Theta_{[(n-1), \ell]}, \Theta_{(n, k)}, \varphi_{\mathrm{hi}}, \varphi_{\mathrm{s}}\right) W\left(\varphi_{\mathrm{hi}}\right) d \varphi_{\mathrm{hi}} \\
\dot{p}_{\mathrm{b}}=\frac{1}{2}\left[\frac{1}{16} \sum_{k=1}^{4} \sum_{\ell=1}^{4} \mathrm{M}_{\varphi_{\mathrm{hi}}}\left\{I_{i}\left(h_{\mathrm{s}}, \Theta_{[(n-1), \ell]}, \Theta_{(n, k)}, \varphi_{\mathrm{hi}}, \varphi_{\mathrm{s}}\right)\right\}+\right. \\
\left.+\frac{1}{16} \sum_{\mathrm{k}=1}^{4} \sum_{j=1}^{4} \mathrm{M}_{\varphi_{\mathrm{hi}}}\left\{I_{q}\left(h_{\mathrm{s}}, \Theta_{[(n-1), \ell]}, \Theta_{(n, k)}, \varphi_{\mathrm{hi}}, \varphi_{\mathrm{s}}\right)\right\}\right]= \\
+\frac{1}{2}\left[\frac{1}{16} \sum_{k=1}^{4} \sum_{\ell=1}^{4} \int_{0}^{2 \pi} I_{i}\left(h_{\mathrm{s}}, \Theta_{[(n-1), \ell]}, \Theta_{(n, k)}, \varphi_{\mathrm{hi}}, \varphi_{\mathrm{s}}\right) W\left(\varphi_{\mathrm{hi}}\right) d \varphi_{\mathrm{hi}}+\right. \\
\left.\sum_{k=1}^{4} \sum_{j=1}^{4} \int_{0}^{2 \pi} I_{q}\left(h_{\mathrm{s}}, \Theta_{[(n-1), \ell]}, \Theta_{(n, k)}, \varphi_{\mathrm{hi}}, \varphi_{\mathrm{s}}\right) W\left(\varphi_{\mathrm{hi}}\right) d \varphi_{\mathrm{hi}}\right]
\end{gathered}
$$

где $W\left(\varphi_{\mathrm{hi}}\right)=\frac{1}{2 \pi}, 0 \leq \varphi_{\mathrm{hi}} \leq 2 \pi-$ плотность вероятности случайной величины $\varphi_{h i}$.

Для получения аналитических выражений вероятностей $\dot{p}_{\mathrm{s}}$ и $\dot{p}_{\mathrm{b}}$ графически построим зависимости индикаторов в интегралах выражений (17) и (18) от переменной $\varphi_{h i}$, которые являются ступенчатыми функциями от этой переменной. Затем перемножим ступенчатые функции, входящие в (17), (18), и вычислим (17), (18) путем представления каждого из слагаемых этих выражений в виде суммы интегралов с интервалами интегрирования, на которых подынтегральные функции не претерпевают скачков. В результате интегрирования получим следующие формулы вероятностей $\dot{p}_{\mathrm{s}}$ и $\dot{p}_{\mathrm{b}}$ :

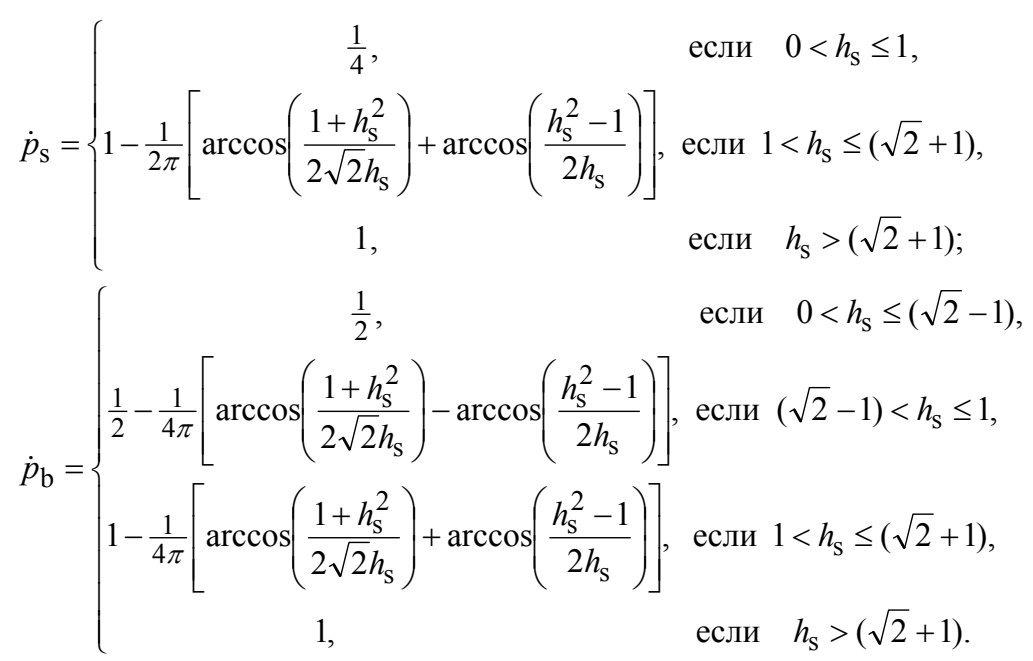

Выражения (19), (20) подтверждают очевидное для некогерентного ПРУ отсутствие зависимости вероятностей $\dot{p}_{\mathrm{s}}, \dot{p}_{\mathrm{b}}$ от начальной фазы сигнала $\varphi_{s}$ для случая воздействия ГП. 


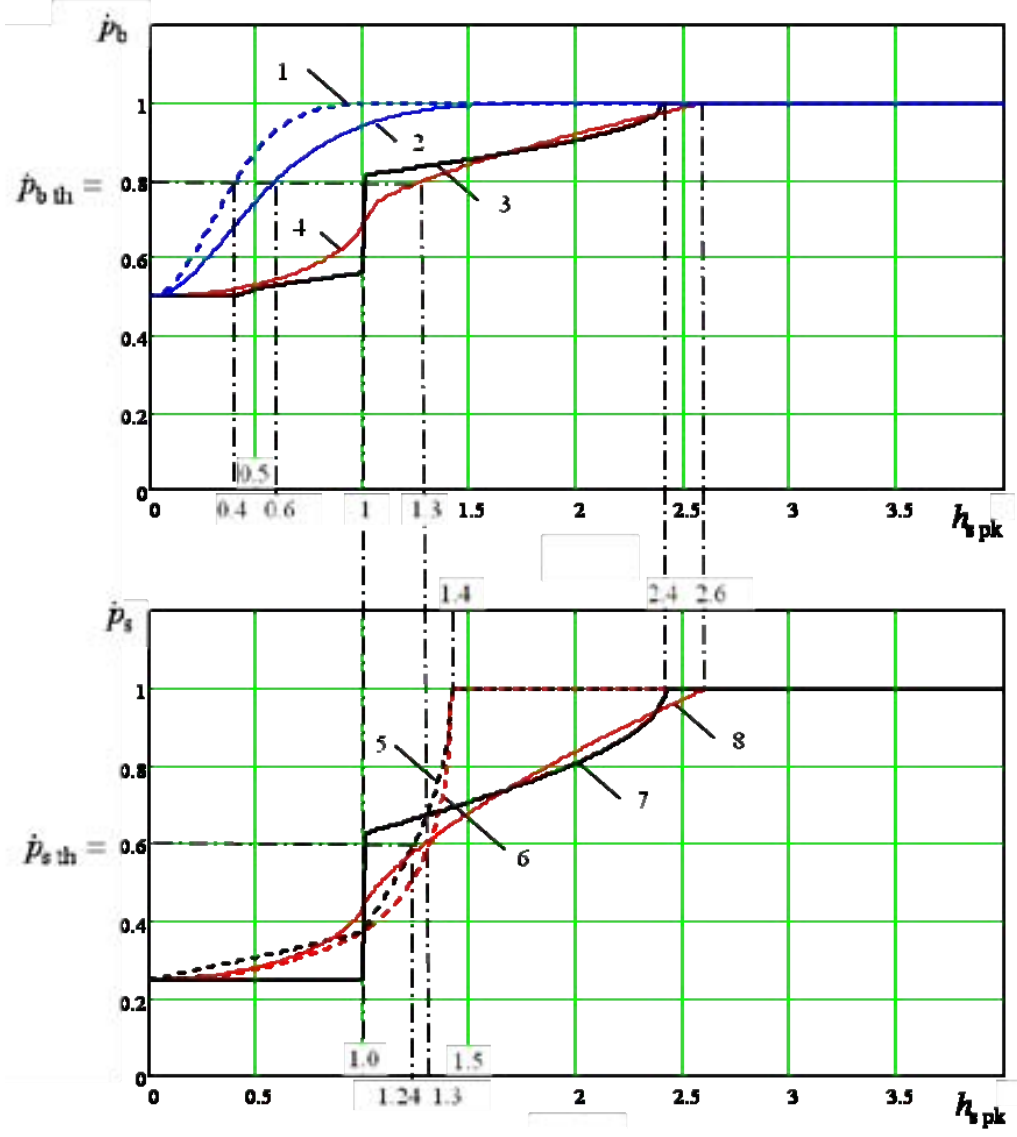

Рис. 1. Зависимости вероятностей $\dot{p}_{\mathrm{b}}$ и $\dot{p}_{\mathrm{s}}$ от $h_{s \mathrm{pk}}$ в некогерентном и когерентном ПРУ ДФРМ сигнала при воздействии фазоманипулированной и гармонической помех: 1 - когерентное ПРУ, гауссовская помеха; 2, 3, 4 - некогерентное ПРУ - гауссовская, гармоническая и фазоманипулированная помехи; 5, 6 - когерентное ПРУ - гармоническая и фазоманипулированная помехи; 7, 8 - некогерентное ПРУ гармоническая и фазоманипулированная помехи

Используя полученные зависимости вероятностей $\dot{p}_{\mathrm{s}}$ и $\dot{p}_{\mathrm{b}}$, проведем анализ помехоустойчивости оптимального некогерентного и когерентного ПРУ ДФРМ сигнала при воздействии фазоманипулированной, гармонической и гауссовской помех по пиковым отношениям сигналпомеха, поскольку у источников станционных и преднамеренных помех пиковая мощность, как правило, ограничена.

Для ФМП и ГП отношение сигнал-помеха по эффективным $h_{s}$ и пиковым $h_{s \mathrm{pk}}$ напряжениям одинаковы, а для гауссовской помехи, пикфактор которой $\Pi_{\mathrm{g}} \approx 3$ при небольших $h_{s}$, справедливо приближенное равенство $h_{\mathrm{s}} \approx\left(\Pi_{\mathrm{g}} / \Pi_{\mathrm{s}}\right) h_{\mathrm{s} \mathrm{pk}}=(3 / \sqrt{2}) h_{\mathrm{s} \text { pk }}$, где $\Pi_{\mathrm{s}}=\sqrt{2}-$ пикфактор сигнала (1).

На рис. 1 по формулам (15), (16), (19) и (20) сплошными линиями построены зависимости вероятностей $\dot{p}_{\mathrm{b}}$ и $\dot{p}_{\mathrm{s}}$ от отношения сигнал-помеха $h_{s \mathrm{pk}}$ по пиковым напряжениям в оптимальном некогерентном ПРУ при воздействии ФМП (2) и ГП (3). Штриховыми линиями построены полученные по методике [4] зависимости вероятности $\dot{p}_{\mathrm{s}}$ от $h_{s \mathrm{pk}}$ в когерентном ПРУ [1, с. 118] при воздействии помех (2) и (3): 
для ФМП

для ГП

$$
\dot{p}_{\mathrm{S}}=\left\{\begin{array}{cc}
\frac{1}{4}+\frac{2}{\pi^{2}}\left[\arcsin \left(h_{\mathrm{s}} / \sqrt{2}\right)\right]^{2}, & \text { если } 0<h_{\mathrm{s}} \leq 1, \\
\frac{1}{2}-\frac{2}{\pi} \arcsin \left(h_{\mathrm{s}} \sqrt{2}\right)+\frac{6}{\pi^{2}}\left[\arcsin \left(h_{\mathrm{S}} / \sqrt{2}\right)\right]^{2}, & \text { если } 1<h_{\mathrm{S}}<\sqrt{2}, \\
1, & \text { если } h_{\mathrm{s}}>\sqrt{2} ;
\end{array}\right.
$$

$$
\dot{p}_{\mathrm{s}}=\left\{\begin{array}{rrr}
\frac{1}{4}+\frac{1}{2 \pi} \arcsin \left(h_{\mathrm{s}} / \sqrt{2}\right), & \text { если } & 0<h_{\mathrm{s}} \leq 1, \\
\frac{5}{2 \pi} \arcsin \left(h_{\mathrm{s}} \sqrt{2}\right)-\frac{1}{4}, & \text { если } 1<h_{\mathrm{s}}<\sqrt{2}, \\
1, & \text { если } \quad h_{\mathrm{s}}>\sqrt{2} .
\end{array}\right.
$$

Для случая воздействия гауссовской помехи на рис. 1 сплошной и штриховой линиями построены зависимости вероятности $\dot{p}_{\mathrm{b}}$ от $h_{s \mathrm{pk}}$ для оптимального некогерентного и когерентного ПРУ соответственно с применением формул [1, ф. (6.125)] и [3].

\section{Обсуждение результатов}

Из рис. 1 видно, что воздействие ФМП и ГП характеризуется пороговым эффектом. Для когерентного ПРУ вероятность $\dot{p}_{\mathrm{s}}$ меньше 1 при $h_{s \mathrm{pk}}$ меньше величины $\approx 1,41$. Применительно к некогерентному ПРУ вероятности $\dot{p}_{\mathrm{b}}$ и $\dot{p}_{\mathrm{s}}$ меньше 1 при $h_{s \mathrm{pk}}$, не превышающих $\approx 2,6$ для ФМП и $\approx 2,41$ для ГП. Следовательно, для достижения порогового эффекта в некогерентном ПРУ требуемая мощность ФМП оказывается в 3,4 меньше, чем для когерентного ПРУ, а мощность ГП - в 2,9 раза.

При отношениях сигнал-помеха по пиковым напряжениям нижеуказанных пороговых значений гауссовская помеха менее неблагоприятна по сравнению с фазоманипулированной и гармонической помехами для обоих рассматриваемых ПРУ.

Анализ приведенных на рис. 1 зависимостей показывает, что для когерентного ПРУ из соизмеримых по эффективности гармонической и фазоманипулированной помех при всех $h_{s \mathrm{pk}}$ более неблагоприятна ФМП. Для некогерентного ПРУ такая однозначность отсутствует. Действительно, для значений $\dot{p}_{\mathrm{b}}$ и $\dot{p}_{\mathrm{s}}$ меньше 1 более неблагоприятной оказывается ГП при $h_{s \mathrm{pk}}$ от 0 до 1 и от $\approx 1,7$ до $\approx 2,37$, а при остальных значениях $h_{s \mathrm{pk}}-$ ФМП.

Для представляющих практический интерес пороговых значений вероятности искажения двоичного элемента $\bar{p}_{\mathrm{b} \text { th }}=0,2$ и вероятности его правильного приема $\dot{p}_{\mathrm{b}}$ th $=1-\bar{p}_{\mathrm{b}}$ th $=0,8$, при достижении которых полностью разрушается информационное содержание передаваемого сообщения [7], наиболее неблагоприятной для некогерентного ПРУ является ФМП. Поскольку требуемое для достижения этого эффекта значение $h_{s \mathrm{pk}} \approx 1,3$, то проигрыш ГП фазоманипулированной помехе по мощности составляет 1,7 раза. Проигрыш гауссовской помехи ФМП по пиковой мощности - 4,9 раза.

Достижение порогового значения вероятности $\dot{p}_{\mathrm{b}}$ th $=0,8$ в когерентном ПРУ ДФРМ сигнала требует в 2,2 раза большей пиковой мощности гауссовской помехи, чем в оптимальном некогерентном ПРУ.

$$
-269-
$$


При указанном $h_{s \mathrm{pk}} \approx 1,3$ из зависимости вероятности $\dot{p}_{\mathrm{s}}$ от $h_{s \mathrm{pk}}$ для ФМП следует пороговое значение этой вероятности $\dot{p}_{\mathrm{s}}$ th $=0,6$. Для достижения этого значения вероятности в некогерентном и когерентном ПРУ требуется практически одинаковая мощность ФМП. Аналогично при воздействии ГП более помехоустойчивым оказывается некогерентное ПРУ, так как для достижения $\dot{p}_{\mathrm{s}}$ th в 1,5 раза больше, чем в когерентном ПРУ.

\section{Заключение}

На основе метода индикаторов решений о переданных элементах получены вероятностные показатели помехоустойчивости оптимального некогерентного ПРУ простого незамирающего ДФРМ-сигнала, а также результаты их сравнения с аналогичными показателями когерентного ПРУ при воздействии фазоманипулированной, гармонической или гауссовской помех. Указанные результаты могут быть использованы для оценки помехоустойчивости ДФРМ-сигнала в условиях воздействия станционных или преднамеренных помех, а также для выбора помех приемно-решающим устройством этого сигнала.

\section{Список литературы}

[1] Окунев Ю.Б. Цифровая передача информации фазомодулированными сигналами. М.: Радио и связь, 1991. 296 с. [Okunev Yu.B. Digital data transmission phase-modulated signals. Moscow, Radio i svjaz', 1991. 296 p. (in Russian)].

[2] Зюко А.Г., Фалько А.И., Панфилов И.П., Банкет В.Л., Иващенко П.В. Помехоустойчивость и эффективность систем передачи информации. М.: Радио и связь, 1985. 272 с. [Zjuko A.G., Fal'ko A.I., Panfilov I.P., Banket V.L., Ivashhenko P.V. Immunity and efficiency of information transmission systems. Moscow, Radio i svjaz', 1985. 272 p. (in Russian)].

[3] Гиршов В.С. Помехоустойчивость когерентного приема многопозиционных сигналов с фазоразностной модуляцией. Радиотехника, 1988, 1, 47-49. [Girshov V.S. Immunity coherent reception of signals from the multi-position phase difference modulation. Radiotehnika, 1988, 1, 47-49 (in Russian)].

[4] Ложкин К.Ю., Поддубный В.Н. Достоверность когерентного приема простых ДФРМ сигналов на фоне помехи произвольной структуры. Радиотехника, 1999, 12, 34-38. [Lozhkin K.Yu., Poddubnyj V.N. Reliability of coherent reception simple DQPSK signals against interference of any structure. Radiotehnika, 1999, 12, 34-38 (in Russian)].

[5] Loeve M. Probability Theory. Princeton, New Jersey, USA: D Van Nostrand, 1955. $685 \mathrm{p}$.

[6] Овчаренко Л.А., Поддубный В.Н. Определение вероятности искажения последовательности двоичных сигналов методом индикаторов решений о переданных элементах. Известия вузов СССР. Серия Радиоэлектроника, 1986, 29 (9), 12-18. [Ovcharenko L.A., Poddubnyj V.N. Determining the probability of distortion sequence of binary signals transmitted by a cell-making indicators. Izvestija Vuzov SSSR. Serija Radiojelektronika, 1986, 29 (9), 12-18 (in Russian)].

[7] Яглом А.М., Яглом И.М. Вероятность и информация. М.: Наука, 1975. 511 с. [Jaglom A.M., Jaglom I.M. Probability and Information. Moscow, Nauka, 1975. 511 p. (in Russian)].

$$
-270-
$$

